\title{
Electrochemical Array Microsystem with Integrated Potentiostat
}

\author{
Jichun Zhang, Yue Huang, Nicholas Trombly, Chao Yang, and Andrew Mason \\ Electrical and Computer Engineering, Michigan State University, East Lansing, MI 48823, USA
}

\begin{abstract}
This paper presents a single-chip electrochemical array system with a high performance CMOS potentiostat suitable for the implementation of a fully integrated instrument-on-chip microsystem. The chip contains a highly sensitive, range-programmable, amperometric readout amplifier that supports cyclic voltammetry assay techniques. Through post-CMOS fabrication, the surface of the chip provides an Au counter electrode, an $\mathrm{Ag} / \mathrm{AgCl}$ reference electrode, and $3 \times 3$ array of individually addressable Au working electrodes. The microsystem is configurable and implements self-correction techniques to provide an adaptive interface for variety of electrochemical and current-mode sensors. The chip supports amperometric output from $10 \mathrm{pA}$ to $10 \mu \mathrm{A}$ and provides good noise performance to achieve subpA resolution.
\end{abstract}

\section{INTRODUCTION}

Electronic measurement of biochemical analyte concentrations is essential for disease diagnose and treatment and the study of biological systems. The standard method for interfacing biological recognition elements (BRE) to electronics utilizes a three-electrode electrochemical measurement technique. The key electronic component for electrochemical measurement is a potentiostat, which can be configured for potentiometric (apply fixed current and measure output voltage) or amperometric (apply fixed voltage and measure output current) readout. Recently, several integrated potentiostats [1-7] have been developed for various applications. For high density electrochemical sensor arrays based on nanostructured biomimetic interfaces [8], the readout electronics must be capable of both high resolution, to exploit the sensitivity of the protein BRE, and large range, to accommodate the variable response of a diversity of BREs. For amperometric detection suitable for this class of sensor, this amounts to sensitivities on the order of picoamperes to nanoamperes over a range from $10 \mathrm{pA}$ to $10 \mu \mathrm{A}$. A low-level current detection circuit with high linearity and high noise performance is required in this approach. Charge integrator based potentiostats that convert currents to a voltage or digital pulse have been utilized in [3, 5, 9]. Although these circuits demonstrate that high resolution can be achieved with charge integrators, the input current range and features provided are not well suited to protein-based electrochemical biosensor arrays.

This paper reports an electrochemical array platform with an integrated potentiostat that features highly sensitive current readout over a wide input range. The chip supports cyclic voltammetry assay techniques and utilized a postCMOS fabrication sequence to form the electrodes of an electrochemical cell directly on the surface of this integrated microsystem.

\section{SYSTEM ARCHITECTURE AND OPERATION}

Figure 1 shows the block diagram of the electrochemical array microsystem. The surface layer of the chip contains a reference electrode, an auxiliary electrode, and a $3 \times 3$ array of working electrodes fabricated on the readout chip using post-CMOS processing. A specific electrochemical cell within the array is selected by an on-chip current switch matrix. The potentiostat block establishes a potential between a selected working electrode and the reference electrode and measures the current flowing between the working and auxiliary electrodes. The circuitry supports both static voltage and cyclic voltammetry assays. The current readout block within the potentiostat converts the current to a voltage using a low-noise, power efficient switched capacitor circuit [11]. The electronics are required to provide low noise, high resolution measurements with high linearity over a wide operation range suitable for various transducers and electrode sizes. The electrodes are required to form a reliable electrochemical cell that can be fabricated post CMOS and maintain compatibility with biological transducer materials.

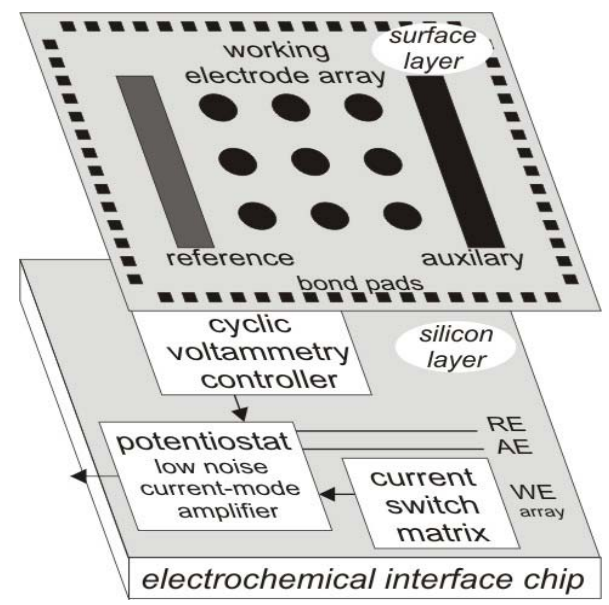

Figure 1. Block diagram of the electrochemical biosensor array microsystem.

The topology of the three-electrode electrochemical microsystem is shown in Figure 2. It consists of a potentiostat biasing structure, an electrode array, a working electrode signal multiplexer, and a single channel current readout circuit. Control signal Vsrc sets the potential applied across the electrochemical cell, and either a constant voltage 
or a sweeping signal necessary for cyclic voltammetry measurements can be applied. Amp2 is configured as a unity gain buffer to establish a potential difference between the reference electrode (RE) and the chosen working electrode (WE). Two resistors and Amp1 form a feedback circuit to mirror the RE potential to the auxiliary electrode (AE). Notice the RE can not sink/source electrical current so all current measured at the WE comes from the $\mathrm{AE}$ to help maintain stability of the RE electrochemical potential.

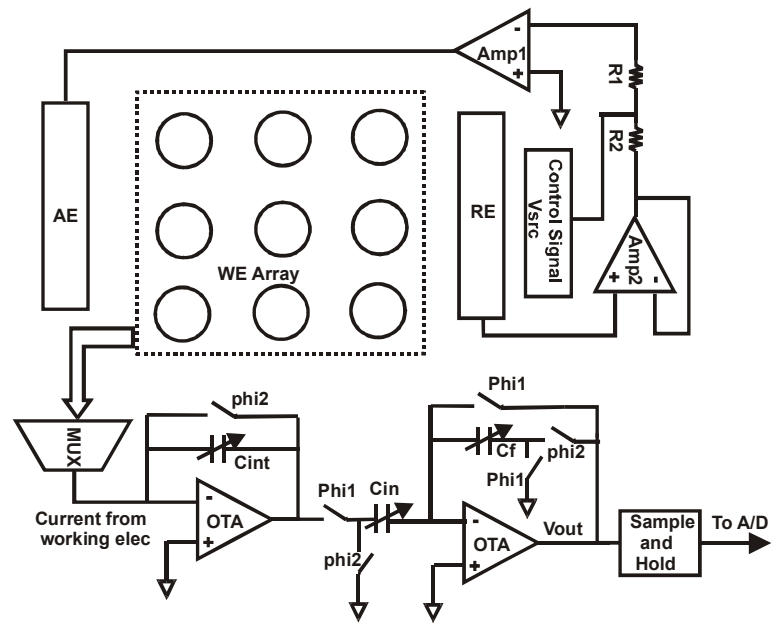

Figure 2. Configuration of the CMOS potentiostat and on-chip electrochemical array.

In the current readout block [11], a switched capacitor (SC) charge integrator converts the sensor output current into a voltage, which then goes through a gain stage that has auto-zero compensation. The output voltage is sampled, held, and then fed to an A/D converter. The entire readout channel utilizes correlated double sampling [10] to reduce the $\mathrm{kT} / \mathrm{C}$ noise due to the feedback capacitor and input capacitor of the programmable gain stage. The output of the highly sensitive current readout block is given by [11]:

$$
V_{\text {out } 1}=\frac{I_{w}}{C_{\text {int }} f_{s}} \cdot \frac{C_{\text {in }}}{C_{f}}
$$

Where, $f_{s}$ is the frequency of phi1, $I_{w}$ is the sensor current from the working electrode, $\mathrm{C}_{\mathrm{int}}$ is the integrator capacitor, $\mathrm{C}_{\mathrm{int}}$ is binary weighted programmable capacitor and $\mathrm{C}_{\mathrm{f}}$ is the feedback capacitor of the programmable gain amplifier (PGA). Equation (1) shows that the circuit can be programmed to operate linearly over different current ranges by adjusting the master clock frequency, the PGA gain, and/or the value of the on-chip programmable integrator capacitor. The circuit has been verified to measure input currents ranging from $10 \mathrm{pA}$ to $10 \mu \mathrm{A}$.

The bias configuration for an electrochemical cell is shown in Figure 3 with the electrical model of an ion channel membrane protein biosensor [8]. $R s$ and $R u$ are the resistances of the solution. $R f w$ represents faradaic resistance, and $C d$ is the double-layer capacitance associated with the working electrode. With a large amplifier gain, the relationship between the voltage across $\mathrm{RE}$ and WE, denoted by $E_{R W}$, and the control signal $V s r c$ is:

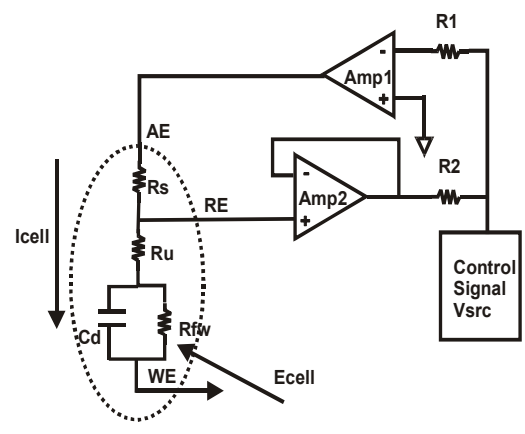

Figure 3. Potentiostat configuration for one electrochemical cell.

$$
\frac{E_{R W}}{V_{s r c}}=\frac{A_{1}\left(R_{u}+R_{f w}\right)}{A_{1}\left(R_{u}+R_{f w}\right)+R_{s}+R_{u}+R_{f w}}
$$

where A1 is the gain of Amp1. When the solution resistance is sufficiently small, the potential across the cell is equal to the applied control voltage, as desired.

Due to the demand for high sensitivity in the current readout circuit, care has been taken to minimize the noise power due to the amplifiers. With the CDS technique, the $\mathrm{kT} / \mathrm{C}$ noise and the clock feedthrough charge can be sampled and subtracted, thereby suppressing noise at the output. Minimum sized complementary CMOS switches have been realized to minimize the charge injection, and dummy switches have been used to reduce clock feedthrough errors. Figure 4 shows that the circuit can resolve low-level currents with high linearity, and noise analysis indicates sub-pA resolution.

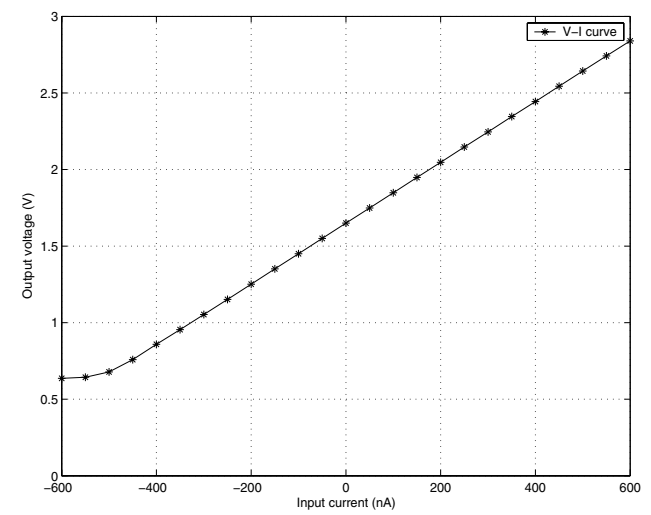

Figure 4. I-V plot of the current readout circuit showing high linearity from -450 to $500 \mathrm{nA}$ at one range setting.

\section{POST-CMOS FABRICATION}

The high sensitivity of the electrochemical readout circuit supports operation with a high density array of microelectrodes. To minimize noise and maximize resolution, the transducers should be directly integrated on the readout chip. A post-CMOS fabrication process has been developed to form an electrode array, including an onchip $\mathrm{Ag} / \mathrm{AgCl}$ reference electrode, on the surface of a CMOS chip. The surface of the chip is cleaned and $20 \mathrm{~nm}$ of $\mathrm{Ti}$ and $100 \mathrm{~nm}$ of $\mathrm{Au}$ are deposited using evaporation. This binds the electrode metals to the CMOS metal layer exposed by openings in the overglass layer created during CMOS 
fabrication. The $\mathrm{Ti} / \mathrm{Au}$ layer is patterned using photolithography and etching solutions to form the working microelectrodes and the larger $\mathrm{AE}$ and $\mathrm{RE}$ electrodes. If an on-chip reference electrode is desired, a $100 \mathrm{~nm}$ layer of $\mathrm{Ag}$ is subsequently deposited and patterned. A spin-on glass is then applied, cured in an oven, and patterned using photolithography and buffered HF. This eliminates difficult alignment of electrode patterns within overglass openings and seals the edges of the electrodes so that only $\mathrm{Au}$ is exposed. If the on-chip RE being fabricated, the chip surface is masked by photoresist and the RE is then exposed to $1 \mathrm{M} \mathrm{FeCl}$, producing an $\mathrm{AgCl}$ layer. This is typically followed by coating Nafion ${ }^{\circledR}$ perfluorinated resin layer over the $\mathrm{RE}$, which is cured in a $120^{\circ} \mathrm{C}$ oven for 60 minutes. Figure 5 illustrates this process.

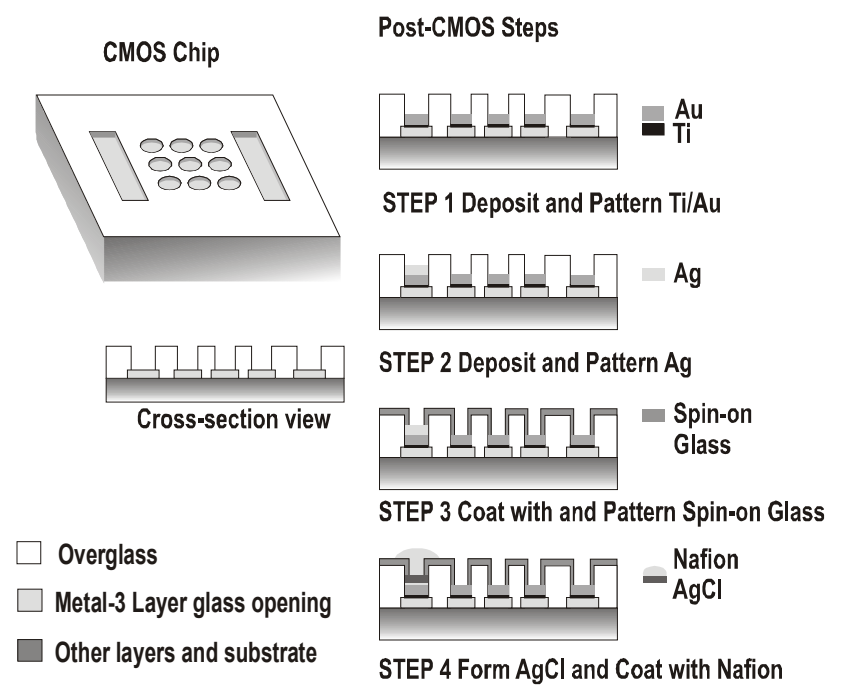

Figure 5. Post-CMOS process flow to form a three-electrode electrochemistry configuration.

\section{MEASUREMENT RESUlts}

The circuit was fabricated in a $0.5 \mu \mathrm{m}$ CMOS process. A die photograph of the $3 \times 3 \mathrm{~mm}^{2}$ chip is shown in Figure 6 . The post-CMOS process sequence described above was performed, and an array of nine $90 \mu \mathrm{m}$ circular working electrodes was formed. The processed die was wirebonded to a PCB, and the chip was quick set epoxy to expose only the on-chip electrodes. The PCB was dipped into the $1 \mathrm{mM}$ potassium ferricyanide (KFCN) solution to perform electrochemical analysis.

Operation of the on-chip three-electrode system was verified by detection of $\mathrm{Fe}^{2+}$ in the KFCN solution using a commercial potentiostat (OMNI-101). Figure 7 shows that the resulting cyclic voltammogram exhibits the characteristics expected from a microelectrode.

A triangle waveform control signal was applied to the on-chip potentiostat, and the resulting RE-to-WE potential was measured. Figure 8 shows the voltage is properly transferred to the electrochemical cell, illustrating that cyclic voltammetry can be performed with on-chip electronics.

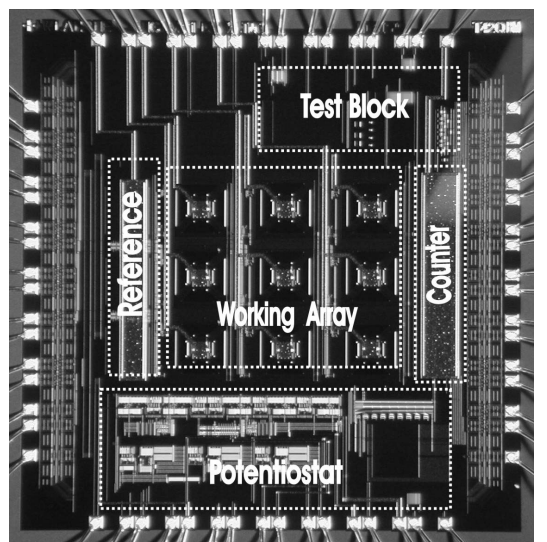

Figure 6 . Die photo of the $3 \times 3 \mathrm{~mm}$ electrochemical array chip.

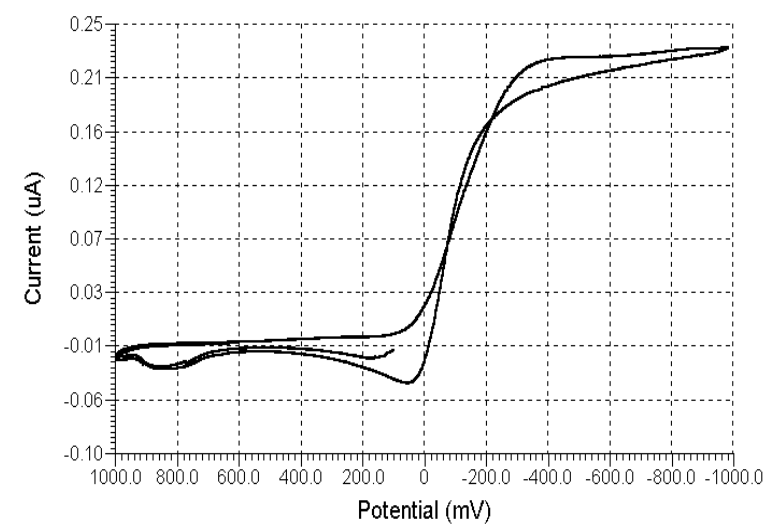

Figure 7. CV plot of on-chip electrode with Omni while the electrochemical array chip dipping in the KFCN solution.

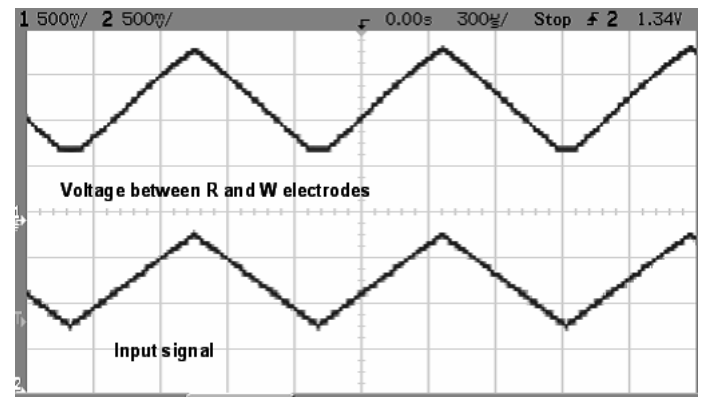

Figure 8. Measured potential between RE and WE (top) and applied control signal waveform (bottom).

The passivated chip was immersed in potassium ferricyanide, and the current from the electrochemical cell was converted to voltage and measured via the on-chip potentiostat. Figure 9 shows the results when a $1 \mathrm{~V}$ (peak to peak) triangle waveform at $2 \mathrm{kHz}$ is applied, similar to a cyclic voltammetry sweep. This figure verifies the functionality of the on-chip potentiostat. A commercial reference electrode was utilized to minimize experimental variables. 


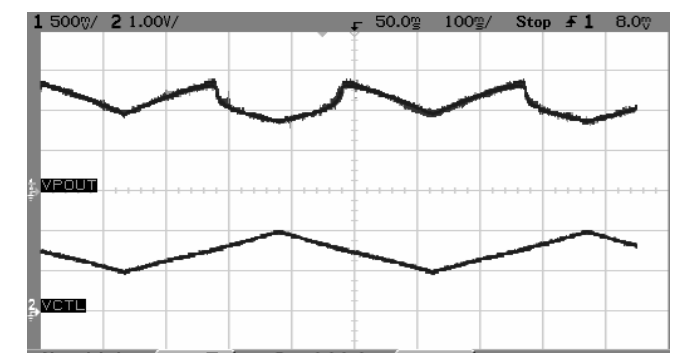

Figure 9. Output of the integrated potentiostat (top) and applied control signal (bottom).

Figure 10 shows the cyclic voltammogram (CV) captured using the on-chip potentiostat and on-chip electrodes (RE was formed on a second chip to minimize packaging complexity). The horizontal axis shows the control voltage, offset by the $1.5 \mathrm{~V}$ analog ground of the chip (with $\mathrm{VDD}=3 \mathrm{~V}$ ). The peak point $0.24 \mu \mathrm{A}$ occurs at the control voltage $1.4 \mathrm{~V}$, which represents about $100 \mathrm{mV}$ potential difference between reference and working electrodes. Due to the manual data collection from on-chip electronics, the sweep rate was only about $0.5 \mathrm{~V} / 30$ seconds, considerably slower than a typical $\mathrm{CV}$ measurement.

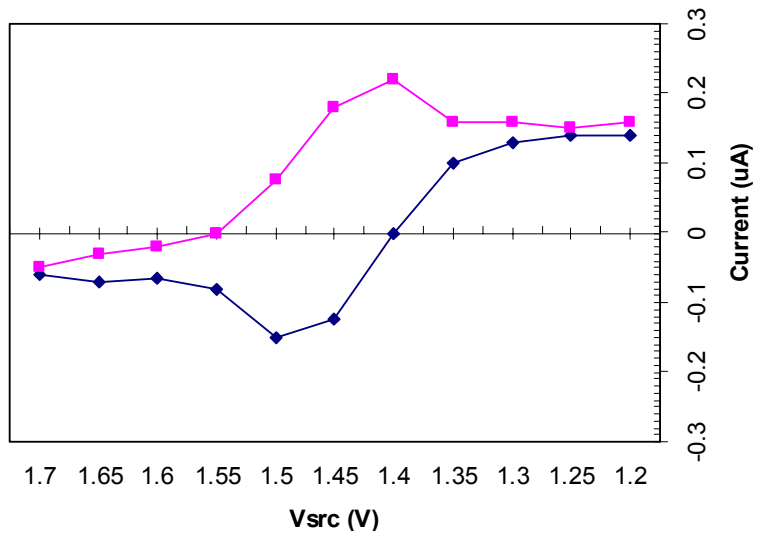

Figure 10. Cyclic voltammogram obtained via the integrated CMOS potentiostat an on-chip electrodes.

\section{CONCLUSION}

An electrochemical array microsystem, with an on-chip potentiostat has been designed. This fully integrated microsystem is tailored to the needs of protein-based biomimetic sensors but applicable to a variety of electrochemical and current-output sensors. The chip supports cyclic voltammetry, provides amperometric readout over a $10 \mathrm{pA}$ to $10 \mu \mathrm{A}$ range, and can resolve subpA currents from a multiplexed array of on-chip working electrodes. Post-CMOS fabrication of electrodes allows transducers to be constructed directly on readout chip to eliminate noise due to interconnects. The microsystem is suitable for a future generation of high-density biosensor arrays.

\section{ACKNOWLEDGMENT}

This work was partially supported by the NSF Center for Wireless Integrated Microsystems (WIMS) under award number ERC-9986866. The authors wish to thank the MOSIS Service for fabrication support.

\section{REFERENCES}

[1] R. F. B. Turner, D. J. Harrison, and H. P. Baltes, "A CMOS potentiostat for amperometric chemical sensors," IEEE JSSC, vol. 22, pp. 473-478, 1987.

[2] R. J. Reay, S. P. Kounaves, and G. T. A. Kovacs, "An integrated CMOS potentiostat for miniaturized electroanalytical instrumentation," in IEEE Int. Solid-State Circuits Conf. (ISSCC) Dig. Tech. Papers, pp. 162-163, 1994.

[3] R. G. Kakerow, H. Kappert, E. Spiegel, and Y. Manoli, "Low-power Single-chip CMOS Potentiostat," Solid-State Sensors and Actuators, and Eurosensors IX. Transducers '95. pp. 142-145, 1995.

[4] A. Frey, M. Jenkner, M. Schienle, C. Paulus, B. Holzapfl, P. Schindler-Bauer, F. Hofmann, D. Kuhlmeier, J. Krause, J. Albers, W. Gumbrecht, D. Schmitt-Landsiedel, and R. Thewes, "Design of an integrated potentiostat circuit for CMOS bio sensor chips," In Proc. IEEE Int. Symposium on Circuits and Systems (ISCAS'03), Bangkok, Thailand, pp. 912, vol.5, 2003.

[5] M. Breten, T. Lehmann, and E. Braun, "Integrating data converters for picoampere currents from electrochemical transducers," In Proc. IEEE Int. Symposium on Circuits and Systems (ISCAS'00), Geneva, pp. 709-712, 2000.

[6] A. Bandyopadhyay, G. Mulliken, G. Cauwenberghs, and N. Thakor, "VLSI potentiostat array for distributed electrochemical neural recording," In Proc. IEEE Int. Symposium on Circuits and Systems (ISCAS'02), pp. 740743, 2002.

[7] S. M. Martin, F. H. Gebara, T. D. Strong, and R. B. Brown, "A Low-Voltage, Chemical Sensor Interface for SystemsOn-Chip: The Fully-Differential Potentiostat," In Proc. IEEE Int. Symposium on Circuits and Systems (ISCAS'04), Vancouver, Canada, pp. IV-892-IV-895, 2004.

[8] P. Kim, N. Kohl, B. Hassler, N. Dotson, A. Mason, R. M. Worden, and R. Ofoli, "An electrochemical interface for integrated biosensors," In Proc. IEEE Int. Conf. on Sensors, pp. 1036-1040 Vol. 2, 2003.

[9] H. S. Narula and J. G. Harris, "VLSI Potentiostat for Amperometric Measurements for Electrolytic Reactions," In Proc. IEEE Int. Symposium on Circuits and Systems (ISCAS'04), Vancouver, Canada, pp. 457-460, 2004.

[10] C. C. Enz and G. C. Temes, "Circuit techniques for reducing the effects of op-amp imperfections: autozeroing, correlated double sampling, and chopper stabilization," Proceedings of the IEEE, vol. 84, pp. 1584-1614, 1996.

[11] J. Zhang, N. Trombly, and A. Mason, "A Low Noise Readout Circuit for Integrated Electrochemical Biosensor Arrays," In Proc. IEEE Int. Conf. on Sensors, Vienna, Austria, pp. 36-39, vol. 1, 2004. 